\title{
关于 VSC 电导测试
}

万梅香 朱道本 李明珠 钱人元

(中国科学院化学研究所, 北京)

在有机导体的电导测量中,一般采用多晶压片的四探针法. 然而, 由于有机导体具有很强 的各向异性, 同时多晶压片晶粒之间存在接触电阻, 而且接触电阻依赖温度而变化, 所以多晶 压片往往不能反映材料真实的电导温度依赖性. 显然, 只有单晶电导才能得出可靠的电导特 性. 可惜要得到足以进行电导测试的单晶往往相当困难. 因此, 在新合成的材料末能得到单 晶之前, 寻找一种方法定性地测出材料电导率的温度依赖性很有必要. 因为电导的温度依赖 性是区分半导体电导和金属电导的重要依据. 1973 年 Coleman ${ }^{[1]}$ 首先提出 VSC 法 (Voltage Shorted Compaction）并用该法测得了 TTF-TCNQ 和 NMP-TCNQ ${ }^{[2]}$ 复合物的电导率的温度 依赖性,其金属一半导体相变温度与单晶得到的结果一致. 但是, 关于 VSC 的实验条件、影 响参数均不清楚. 我们对 VSC 法进行了一些研究并提出 VSC 法的一个物理模型, 能较好地解 释实验中所观察到的现象. 在这个基础上对一些新合成的有机导体进行了 VSC 法电导测量.

\section{实 检方 法}

多晶压片是在 $7000 \mathrm{~kg} / \mathrm{cm}^{2}$ 的压力下将多晶材料压制成直径为 $7 \mathrm{~mm}$ 的圆片, 然后用刀片 切割成矩形样品, 四根直径为 $20 \mu \mathrm{m}$ 的金丝用银胶粘在样品上, 用四探针法测量电导. VSC 法 是在多晶压片的基础上, 用导电银胶将电压端短路, 测试器件的结构如图 1 所示. 电导率的温 度依赖性是将样品放人自制的低温装置，温度用铂电阻温度计通过 $8024 \mathrm{~A}$ 型数字万用表测量. 从 1,4 端输人恒定电流 $I(1 \sim 50 \mu \mathrm{A})$, 用 148 型毫微伏计测量 2,3 端的电位降 V.

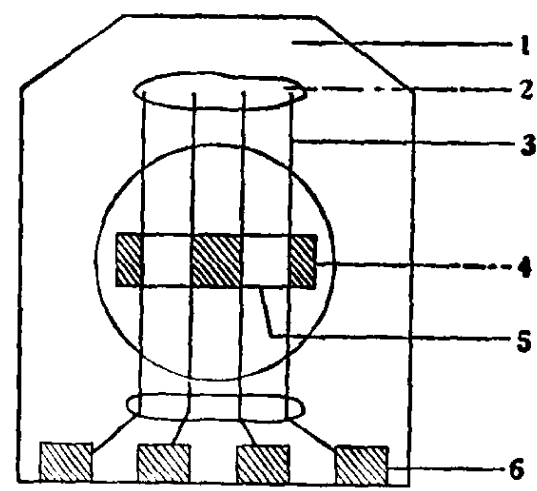

图 1 VSC 器件的结构示意图

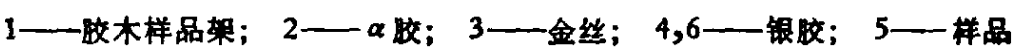




\section{实验结果和讨论}

VSC 电导值与短路电阻的关系 VSC 法与普通四探针法唯一的区别是 2,3 端被银 胶短路了. 因此, 短路电阻对 VSC 电导的影响是很重要的. 我们以 DIPS $\phi_{4} \cdot(\mathrm{TCNQ})_{2}$ (分子 式见后)为对象, 研究了短路电阻对该复合物的 VSC 电导的影响. DIPS $\phi_{4} \cdot(\text { TCNQ })_{2}$ 的 VSC 器件在氩气保护下于 $50^{\circ} \mathrm{C}$ 热处理 2 小时之后再进行电导测量. 其实验结果如图 2 所示. 可

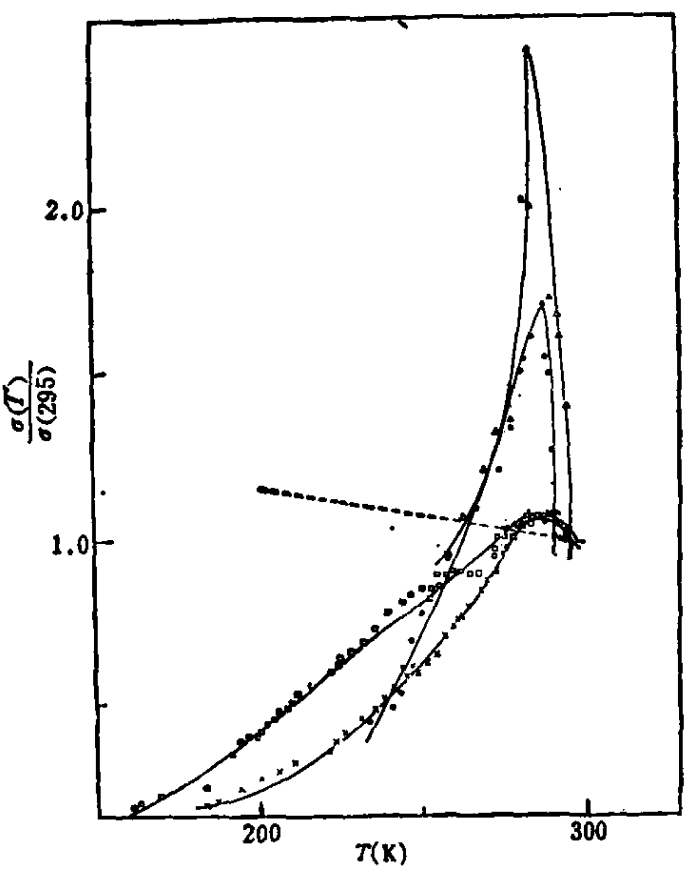

图 2 vSC 电导的淈度依赖珄与短路电阻关系 $\times 2 \mathrm{~K} 0 ;-1250 ; \Delta 0.50$; 口多晶;……银胶
清楚地看出, DIPS $\phi_{4} \cdot(\mathrm{TCNQ})_{2}$ 的 VSC 电导蜂值 高度随短路电阻的减少而增加, 但峰值位置基本 不变.

VSC 电导出现银胶电导的条件 VSC 法 的特点是用银胶短路了电压端.因此, 首先要知道 银胶电导特性. 银胶电导是在聚四偂乙烯样品支 架上用标准的四探针法测量银胶的电导率的温度 依赖生. 如图 3 上线所示的结果表明银胶电导随 温度的降低而缓慢地增加, 呈现典型的金属电导 特性. 我们观察到在 VSC 器件制备不当时, VSC 器件会出现银胶电导. 我们以 (Ad) (TCNQ) 2 为 对象, 发现 $D=R_{14} / R_{23}=10,55,100$ 时 (Ad) (TCNQ) ${ }_{2}$ 的 VSC电导的温度依赖性如图 3 下线所 示的直线与银胶电导相似. 但是, 当 $D=900$ 时, (Ad) (TCNQ $)_{2}$ 的 VSC 电导才定性地模拟了单晶 的电导率温度依赖性,如图 4 所示. 要使 VSC 法 成功必须在多晶压片的 2,3 端靠近样品表面附近

\section{一层粒间的接触电阻完全被银胶短路了.}

VSC 电导与单昆电导的温度依赖性的比较我们比较了 (Ad) (TCNQ) 2 复合物的 VSC 电导与单晶电导的温度依赖性测试结果. (Ad) (TCNQ) 2 单晶是用电化学方法从乙腈和 水的溶液中生长的, 得到 $1.8 \mathrm{~mm} \times 0.05 \mathrm{~mm} \times 0.05 \mathrm{~mm}$ 的完整单晶. 该晶体的室温电导率 $\sigma_{R T}-2.9 \times 10^{2}(\Omega-\mathrm{cm})^{-1}$. (Ad) $(\mathrm{TCNQ})_{2}$ 的 VSC 电导率和单晶电导率的温度依赖性的比

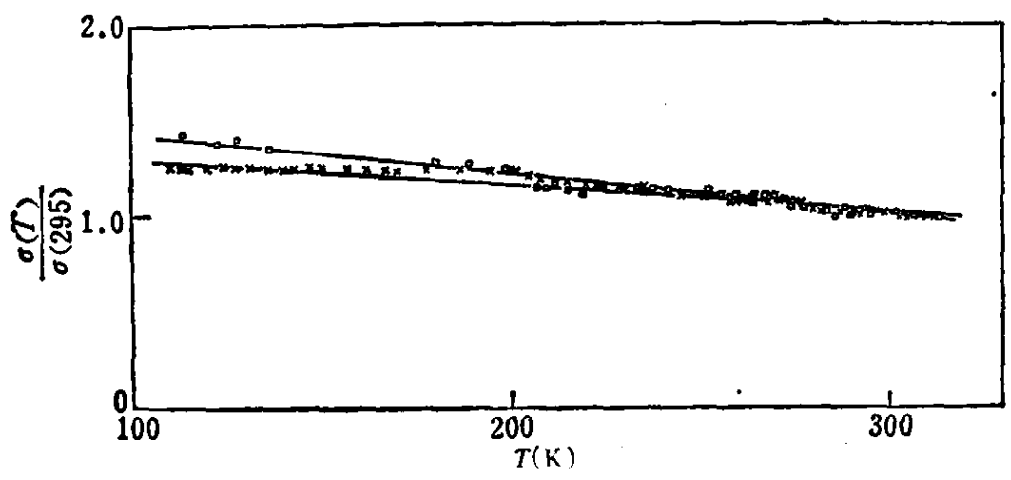

图 3 VSC 电导出现银胶电导的条件

上线一银胶; 下线- $D=10,55,100$ 的 (Ad) $\mathrm{TCNQ}_{2}$ 的 VSC 电导 


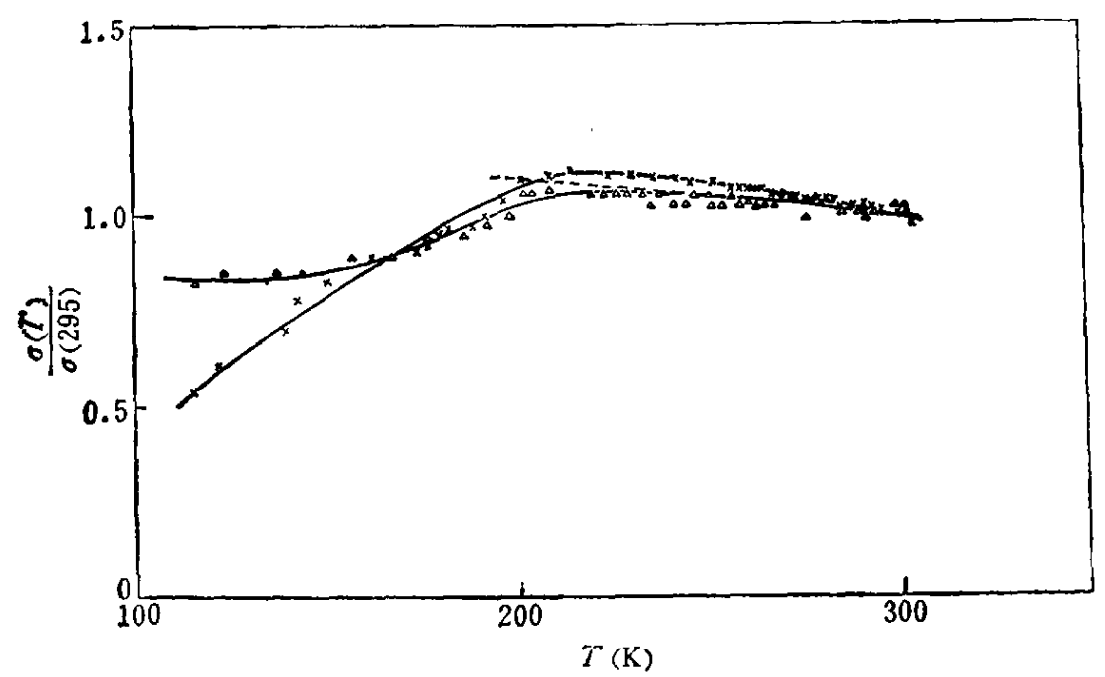

图 4 (Ad) (TCNQ) 2 的单晶和 VSC 电导率的温度依赖性的比较 $\times$ 单晶; $\triangle \mathrm{VSC}$

较如图 4 所示, 其 $\mathrm{T}_{c}=210^{\circ} \mathrm{K}$ 左右, 与 $\mathrm{Kobayash}^{\left[{ }^{[4]}\right.}$ 从晶体结构分析得到的 $\mathrm{T}_{c}=200^{\circ} \mathrm{K}$ 相接 近, 但与 Буравов ${ }^{[4]}$ 等的结果不一致.

一些新合成的有机导体的 VSC 电导测试 我们实验室合成的一些 TCNQ 复合物有 机导体 ${ }^{[5]}$ 的 VSC 电导测试结果如图 5 所示. DIPS $\phi_{4} \cdot(T C N Q)_{2}$ 属于具有高、窄的比电导峰值的 第 I 类有机导体，而 DIPS $\phi_{4} \cdot$ TCNQ，(Ad)(TCNQ) ${ }_{2}$ 和 (TMAAd) (TCNQ) 2 是属于具有宽、弱 比电导峰值的第 II 类有机导体，而 (DAd) (TCNQ) 4 是典型的第 III 类半导性有机导体.

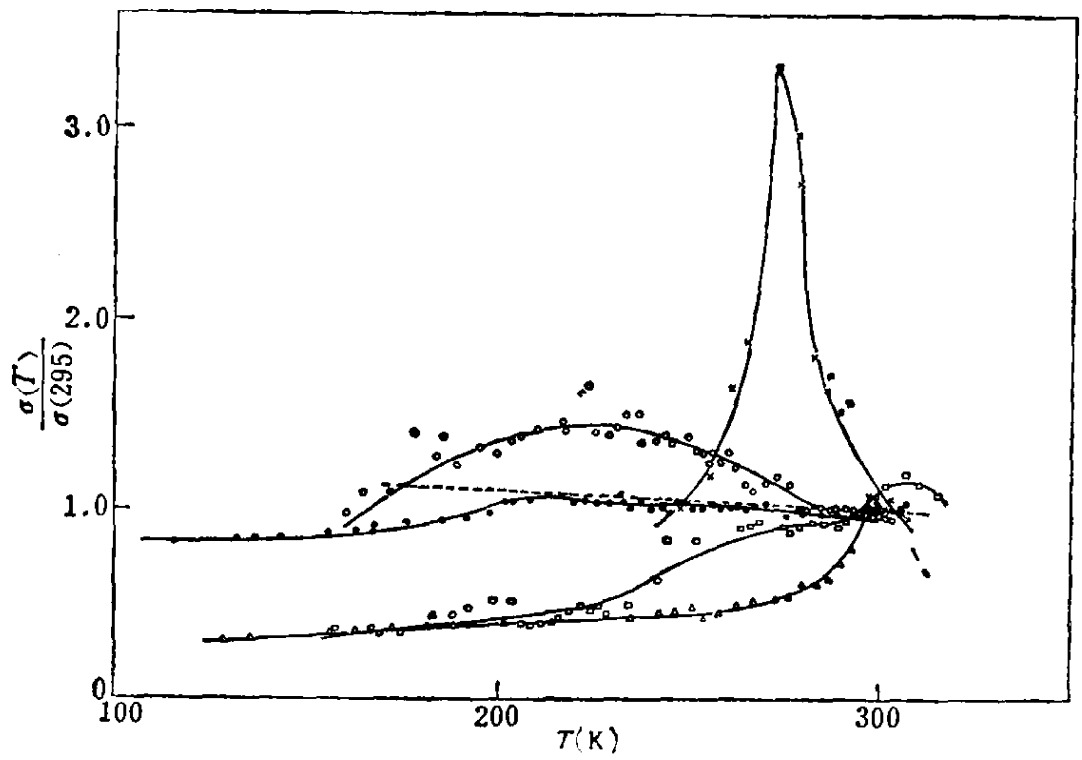

图 5 一些新合成的有机导体的 VSC 电导率的㴜度依赖性 $\times \operatorname{DIPS}_{4} \cdot \mathrm{TCNQ}_{2}$

- (Ad)(TCNQ) $;$ O DIPS $\phi_{4} \cdot \mathrm{TCNQ}$; 结构

名称

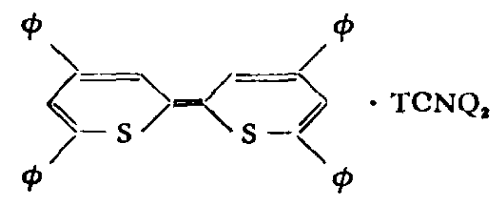

$\operatorname{DIPS} \phi_{4} \cdot(\text { TCNQ })_{2}$ 


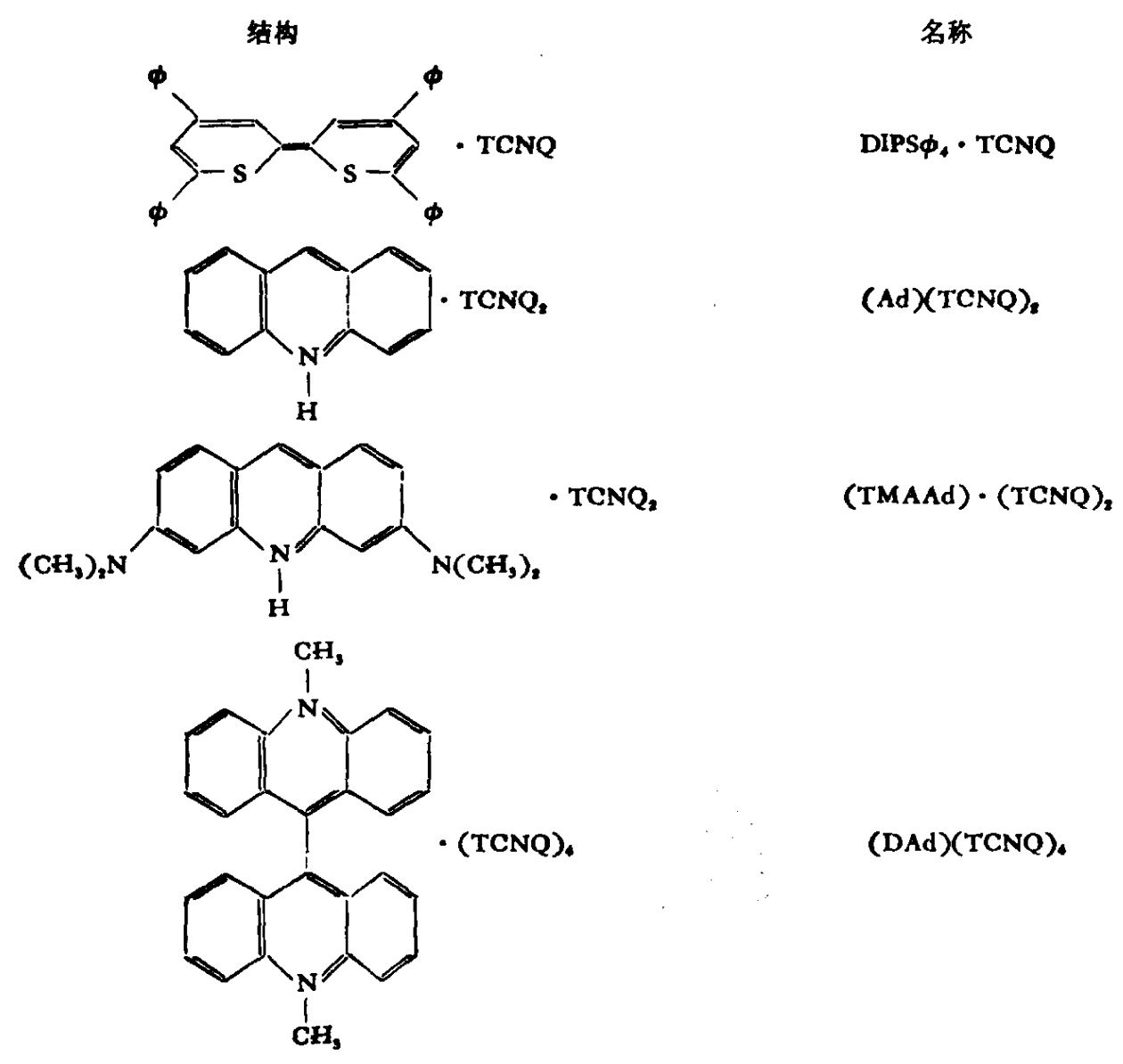

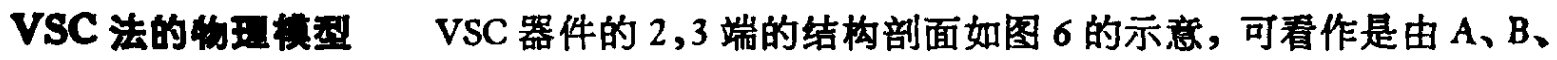
C 三层组成. A 层完全由银胶组成, B 层是有机导体晶粒完全浸在导电银胶中, 晶粒间的接触 电阻完全被银胶所短路, 该层的电阻值可看成是试样的电阻与银胶电阻的串联值, $\mathrm{C}$ 层是有机 导体本身是材料的电阻与晶粒间接触电阻的串联值. 因此, VSC 器件 2,3 端的电阻应看成是 $A 、 B 、 C$ 三层电阻的并联.

当恒定电流 I 通过 VSC 器件时, A、B、C三层起分流作用. 一般晶粒间的接触电阻最大, 银胶电阻最小, 在 2,3 端的电位降只需考虑 $A 、 B$ 层的并联已是足够的近似. 假设 2,3 端之 间的距离为 1 , 样品的宽度为 $a, A 、 B$ 层的厚度分别为 $\mathrm{d}_{1}$ 和 $\mathrm{d}_{2}$ 以及试样、银胶和 VSC 器件的电 导率份别为 $\sigma_{\mathrm{S}} 、 \sigma_{\mathrm{A}}$ 和 $\sigma_{\mathrm{vSC}}$, 则

$$
\frac{I}{V}=\sigma_{\mathrm{VSC}} \frac{a\left(d_{1}+d_{2}\right)}{l} \doteq \sigma_{\mathrm{Ag}} \frac{a d_{1}}{l}+\frac{1}{\frac{\phi_{\mathrm{S}}}{\sigma_{\mathrm{S}}}+\frac{\phi_{\mathrm{Ag}}}{\sigma_{\mathrm{Ag}}}} \cdot \frac{a d_{2}}{l},
$$

或

$$
\begin{aligned}
& \sigma_{\mathrm{Vsc}}(T)-\frac{d_{1}}{d} \sigma_{A g}(T)\left\{1+\frac{d_{2}}{d_{1}}\left(\frac{1}{\phi_{A g}+\frac{\sigma_{A g}(T)}{\sigma_{S}(T)} \phi_{S}}\right)\right\}, \\
& d=d_{1}+d_{2}
\end{aligned}
$$

式中 $\phi_{A g}$ 和 $\phi_{S}$ 分别为 $B$ 层中的 $A g$ 胶和待测材料的体积分数. 因此当 $\phi_{A g} \ll \phi_{S}, \sigma_{S} \ll \sigma_{A g}$ 时, 则 $\phi_{\mathrm{AB}} \ll \frac{\sigma_{\mathrm{Ag}}}{\sigma_{\mathrm{B}}} \phi_{\mathrm{S}}$, 故(2)式可近似表示如下:

$$
\sigma_{\mathrm{vsc}}(\mathrm{T}) \simeq c_{1} \sigma_{\mathrm{Ag}}(\mathrm{T})+c_{2} \sigma_{\mathrm{S}}(\mathrm{T}),
$$




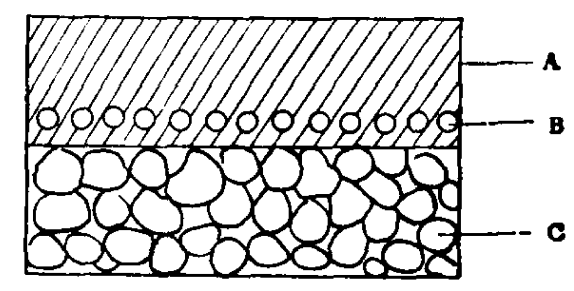

图 6 VSC 器件的 2,3 端的剖面结构示意图 A一一银胶层; $\mathrm{B}$-一浸涌层; $\mathrm{C}$ 一一有机导体层

其中

若将(3)式对温度微分得:

$$
\left\{\begin{array}{l}
c_{1}=\frac{d_{1}}{d_{1}+d_{2}}, \\
c_{2}=\frac{d_{2}}{d_{1}+d_{2}} \cdot \frac{1}{\phi_{s}},
\end{array}\right.
$$

$$
\frac{d \sigma_{\mathrm{VsC}}}{d \mathrm{~T}} \doteq c_{1} \frac{d \sigma_{A R}(\mathrm{~T})}{d \mathrm{~T}}+c_{2} \frac{d \sigma_{\mathrm{S}}(\mathrm{T})}{d \mathrm{~T}}
$$

因为 $d \sigma_{\mathrm{Ag}} / d T$ 值很小并接近为常数, 故 VSC 电导率的温度依赖性完全由试样电导率的温度依 赖性所决定,而 VSC 电导值本身是没有意义的.

同样由(4)式看出, 要使 VSC 电导的温度依赖性更能反映试样电导率的温度依赖性则要 求浸润层 (B 层) 的厚度 $d_{2}$ 要大, 而银胶层 (A 层) 的厚度 $d_{1}$ 要小, 此时 (4)式中第二项的贡献增 大.

致唃: 本所慈守代同志协助建立低温测试设备,特此致谢。

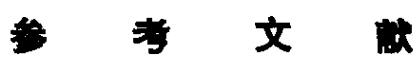

[1] Coleman, L. B., Cohen, M. J., Sandman, D. J., Yamagishi, E. G., Garito, A. F. \& Heeger, A. J., Solid State Commun., 12(1973), 1125.

[2] Coleman, L. B., Rev. Soi. Lnstrum., 49(1978), 58.

[3] Kobayashi, H., Bull. Chem. Soc. Japan, 47(1974), 1346.

14 Ј Буравов Л. И., Фебутин Д. Н. и Щеголев И. Ф., Ж. Эксп. Теор. Физ., 59(1970), 1125.

５］朱道本、万梅香、本明珠、过丁、钱人元, Mol. Cryst. \& Liq. Cryst- (将发表). 